УДК 539.376

\title{
Translation-rotation plastic flow in polycrystals under creep
}

\author{
V.E. Egorushkin ${ }^{1}$ and V.E. Panin ${ }^{1,2}$ \\ ${ }^{1}$ Institute of Strength Physics and Materials Science SB RAS, Tomsk, 634055, Russia \\ ${ }^{2}$ National Research Tomsk Polytechnic University, Tomsk, 634050, Russia
}

\begin{abstract}
Grain boundary sliding is the primary process of plastic flow in polycrystals under creep which is accommodated by multiscale translation-rotation modes of intragranular plastic deformation. The steady stage of creep is characterized by constant creep rates which is related to slow rotation of sliding dislocations and material mesofragmentation. The rate of the tertiary creep is being increased which is related to the similarity of grain boundary sliding and plastic flow within near-boundary zones, where high lattice curvature arises. Here we develop a nonlinear theory of micro, meso- and macroscale plastic flow and propose a constitutive equation for its velocity, rotation modes, local irreversible stress, temperature, and heat and mass flux densities with regard to structural transformation. The theory agrees well with experimental data on multiscale translation-rotation in Al polycrystals under creep, suggesting that fracture in tertiary creep is caused by a multilevel increase in lattice curvature in a deformable polycrystal. Fracture is initiated at the interface of grain boundary sliding and near-boundary regions where lattice curvature results in cracks.
\end{abstract}

Keywords: nonlinear theory of plastic flow, structural transformation, tertiary creep, multiscale rotations

DOI $10.24411 / 1683-805 X-2018-13003$

\section{Трансляционно-ротационное пластическое течение поликристаллов при ползучести}

\author{
В.Е. Егорушкин ${ }^{1}$, В.Е. Панин ${ }^{1,2}$ \\ ${ }^{1}$ Институт физики прочности и материаловедения СО РАН, Томск, 634055, Россия \\ ${ }^{2}$ Национальный исследовательский Томский политехнический университет, Томск, 634050, Россия
}

В условиях ползучести поликристаллов зернограничное скольжение является первичным процессом пластического течения, которое аккомодируется многоуровневыми трансляционно-ротационными модами внутризеренной пластической деформации. Стадия установившейся ползучести развивается с постоянной скоростью, что обусловлено медленными ротационными модами внутризеренного дислокационного скольжения и мезофрагментации материала. Скорость ползучести на третьей стадии резко возрастает, поскольку ротационные моды зернограничного скольжения и приграничного пластического течения, где возникает кривизна решетки, становятся близкими. В настоящей работе приведены основы нелинейной многомасштабной теории ползучести и предлагается определяющее уравнение для описания связи со структурными трансформациями скорости ползучести, ротационных мод, локальных необратимых напряжений, температуры, плотности потоков тепла и массы. Наблюдается хорошее согласие теории с экспериментальными данными для ползучести поликристаллов высокочистого Al. Разрушение на третьей стадии ползучести связано с многоуровневым возрастанием кривизны решетки в деформируемом поликристалле. Зарождение разрушения происходит на интерфейсе зернограничного скольжения и приграничных зон зерен, где сильная кривизна решетки вызывает генерацию трещин.

Ключевые слова: нелинейная теория пластического течения, структурная трансформация, третья стадия ползучести, многоуровневые ротации

\section{Introduction}

Creep in polycrystalline materials at constant applied stress is a multiscale process behind which lie translationrotation modes of plastic deformation and fracture. The process is guided by grain boundary sliding as a source of primary plastic rotations which are accommodated, accord- ing to the angular momentum conservation law, by multiscale intragranular rotations. Creep provides intriguing patterns of grain boundary sliding because grain boundaries lack translation invariance and triple grain junctions form a stochastic system of internal stress concentrators [1-9]. 
It is commonly supposed that high strain rates early in creep result from stress relaxation at triple junctions whose boundaries generate nonlinear defects as sources of intragranular dislocation deformation. Next comes steady creep in which point defects in grain boundaries create a steady system of positive ion clusters from which dislocations develop to accommodate intragranular strains. This stage of creep features low rates because of easy rotations at grain boundaries [10] and slow accommodating rotations inside grains, which determines actual rotational modes of polycrystalline materials under creep. Finally, the creep rate increases steeply up to the point of fracture $[3,4,9]$. The question of what mechanism accelerates the creep at its final, tertiary stage is debatable but it is quite clear that all stages of creep involve multiscale translations-rotations and that their effect at the tertiary stage should be strong to eventually culminate in plastic collapse. The foregoing makes it worthy to elucidate what lies behind rotational deformation in polycrystals under creep.

One of the concepts suggests that plastic rotations in solids are much contributed by lattice curvature $[3,4,10]$. This concept, as applied to strength and plasticity, has been discussed in many papers [11-18] but clarifying the relationship between lattice curvature and rotation modes of plastic flow requires a theoretical description and experimental verification. Here we provide such a description and its experimental verification on the example of plastic flow and fracture in polycrystals under creep, supporting the view that any fracture in a deformed solid represents rotation modes and so does tertiary creep.

\section{Theoretical approach and verification method}

Our theoretical description of nonlinear plastic flow and its translation-rotation modes is based on the following concept. Unlike elastic equilibrium described by elastic wave equations and Hooke's law (equation of state), plastic equilibrium needs equations descriptive of elastic discontinuities, their density and flux $[19,20]$. The constitutive equation of plastic state is determined by positive entropy production via defect and energy flows escaping from deformed regions and responsible for local strain irreversibility. Plastic displacements are a sort of compensating fields for elastic strains in a continuous medium, and their dynamics can be described by gauge fields or curved spacetime geometry. The idea of using gauge fields or Riemann geometry to describe defects in a deformed solid dates back to the 1980s [21-23] but it has not been advanced since then. Attempts to explain macroscopic plastic deformation in terms of microscopic or atomic motion are successful only if we consider model specimens with low defect (dislocation) densities and fail if we consider real materials. The escape of a defect from a strain localization zone is just a driving force for entropy production and irreversibility rather than an event responsible for macroscopic deformation.
Considering structured media on meso- and macroscales in continuum mechanics and electrodynamics, we are to average microscale equations, and in dislocation physics, such averaging leads to dislocation polarization [22, 23]. Electrodynamics considers a medium and a magnetic field as two different objects for which a field-averaged problem is formulated. Plasticity physics and mechanics treat a medium and defects as a single object and needs averaging over its scale. One of the averaging procedures uses scale transformation and $\varepsilon$ extension [24], making it possible to average microscale equations and to derive their macroscale analogues with extensions descriptive of nonlinear plastic waves in a deformed solid [16, 19, 20]. Such a nonlinear wave is basic in our analysis of deformation processes, allows their description from a single standpoint, and provides the way to better understand the origin of translationrotation plastic flow.

The method of experimental verification consists in analyzing the processes occurring in high-purity (99.999 at \%) A999 Al polycrystals under creep at ambient temperature. When creep is investigated at $50^{\circ} \mathrm{C}$, this type of polycrystals can experience highly pronounced grain boundary sliding and attendant strong plastic rotations on meso- and macroscales. According to the angular momentum conservation law, the structure of the material is involved in intragranular deformation which is accommodating process by multiscale rotations of opposite sign.

For our verification, we used flat dumbbell-shaped specimens with a gage section of $1.2 \times 8 \times 37 \mathrm{~mm}$ cut from rolled sheet and annealed at $513 \mathrm{~K}$ for $0.5 \mathrm{~h}$ such that the average grain size was $d=400 \mu \mathrm{m}$; for structural analysis, the specimen surface was electrolytically polished.

The specimens were tested for creep under uniaxial tension at a stress of 11,13 , and $18 \mathrm{MPa}$ and temperature of $323 \mathrm{~K}\left(0.35 T_{\mathrm{m}}\right)$. The elongation was measured with a dial indicator accurate to $\pm 1 \mu \mathrm{m}$. The structure of the material at different creep stages was analyzed by optical microscopy (Axiovert 25CA), interference microscopy (NewView 6200), transmission electron microscopy (JEOL 2100), and scanning electron microscopy (Quanta 2003D).

\section{Results}

\subsection{Plastic strain waves}

Let us analyze the dynamics of deformation in an elastoplastic medium using a macroscale plastic equilibrium equation which relates the flux and density of defects (discontinuities of displacement vector fields), elastic stresses, and potential external sources of plastic strains $[14,15,20]$ :

$$
\varepsilon_{\mu x \delta} \partial_{x} \alpha_{\delta}^{\beta}=\frac{1}{c^{2}} \partial_{0} J_{\mu}^{\beta}+\sigma_{\mu}^{\beta}-\frac{c_{\mu \nu}^{\beta \gamma}}{E} p_{\gamma}^{\nu},
$$

where $\varepsilon_{\mu x \delta}$ is the Levi-Civita symbol, $\partial_{x}$ is the derivative with respect to space variables, $\partial_{0}$ is the time derivative with summation over reiterating indices, $\alpha_{\delta}^{\beta}$ is the linear defect density tensor (for dislocations if the discontinuity 
is a multiple of the lattice constant), $J_{\mu}^{\beta}$ is the plastic flux or defect flux density, $\sigma_{\mu}^{\beta}=c_{\mu \nu}^{\beta \gamma} / E \partial_{\gamma} \ln u_{v}$ are local elastic stress concentrators determined by Hooke's law, $c_{\mu \nu}^{\beta \gamma}$ are elastic moduli, $E$ is Young's modulus, $c$ is the velocity of sound, and $p_{\gamma}^{v}$ is plastic distortion. The plastic distortion $p_{\gamma}^{v}$ is of dual nature: in combination with $\alpha$ and $J$, it determines the current strain, and in combination with $\sigma_{\mu}^{\beta}$, it can represent external strain-stimulating sources.

Generally, it is impossible to discard $p_{\gamma}^{v}$ from Eq. (1) in a way similar to that in electrodynamics because gauge conditions are specified by specific problem conditions.

For the elastic sources determined by the scalar $\psi(x, t)$ rather than by the displacement vector (volume fraction of deformed material), Eq. (1) takes the form

$$
\varepsilon_{\mu x \delta} \partial_{x} \alpha_{\delta}=\frac{1}{c^{2}} J_{\mu}+\sigma_{\mu}-c_{\mu \nu}^{\gamma} p_{\gamma}^{v}
$$

where $\sigma_{\mu}=c_{\mu}^{\gamma} \partial_{\gamma} \ln \psi(x, t), c_{\mu}^{\gamma}=\sum_{\beta, v} c_{\mu \nu}^{\beta \gamma} / E$. For certain loading conditions, Eq. (1) can also be given in vector form.

Deriving macroscale relations requires scale averaging of Eqs. (1), (2) for which we can use scale transformation and $\varepsilon$ extension that gives us the plastic equilibrium equation for macro- and mesoscales [20]:

$$
V_{\mu}=\varepsilon_{\mu r \delta} \partial_{x} \Phi_{\delta}-c_{\mu \nu}^{\gamma} P_{\gamma}^{v} .
$$

Here,

$$
\Phi=-c^{2} \int \alpha \mathrm{d} t, V=-J, P_{\gamma}^{v}=c^{2} \int p_{\gamma}^{v} \mathrm{~d} t, c_{\mu \nu}^{\gamma}=\sum_{\beta}\left\langle c_{\mu \nu}^{\beta \gamma}\right\rangle / E,
$$

where $\left\langle c_{\mu \nu}^{\beta \gamma}\right\rangle$ is the space average derived elsewhere [20] and the time average is over an observation period. The opposite signs of $\Phi$ and $V$ indicate that these are mass parameters rather than defect characteristics.

The averaged equations provide the following valuable data. First, meso- and macroscale plastic deformation, like microscale deformation [25], develops for no change of volume because such changes vanish during scale averaging (volume conservation law). Second, we have an elastic stress concentrator after averaging, which also provides similarity between macro- and microscale equations, in particular (1), except only for variations in averaged physical parameters (elastic moduli, density, etc.). Third, straininduced defects are responsible for instantaneous plastic flow velocities. Thus, scale similarity holds for plastic deformation.

The above conclusions follow from nonlinear equilibrium and from any local solutions of its equation. Because plastic deformation is actually the evolution of local displacement fields and their discontinuities, we need solutions of (2), (3) for a local region rather than for the whole coordinate space to trace the behavior of a plastic strain wave as a certain space curve in this region. The theory of space curves is based on the Frenet equation in a local coordinate system, allowing us to obtain nonlinear equations of motion solvable by the inverse scattering method [20].
The equations for localized plastic strain (2), (3) show that deformation develops as nonlinear spiral waves the characteristics of which are determined by the geometry of a deformed region (bending-torsion), its size (length, lateral dimension), and physical parameters (elastic modulus, density) [20]. Reasoning that plastic strain waves are a fundamental phenomenon of macro-, meso-, and microscales, let us consider these equations and their solutions at greater length as they allow us to better understand how such waves contribute to plastic and other types of deformation, to analyze differences in plastic flow on different scales (up to nano), and to link the physics of defects and the mechanics of plastic deformation.

Following the method proposed elsewhere [20], we can represent the term $c_{\mu \nu}^{\gamma} p_{\gamma}^{v}$ as the sum of the rotor of a certain vector $\boldsymbol{\Phi}$ and the scalar gradient $f$ :

$$
c_{\mu \nu}^{\gamma} p_{\gamma}^{v}=\varepsilon_{\mu x \delta} \partial_{x} \Phi_{\delta}^{\prime}+\partial_{\mu} f
$$

and take the rotor from both parts of Eq. (3). Then, (3) assumes the form

$$
\varepsilon_{n k \mu} \varepsilon_{\mu r \delta} \partial_{k} \partial_{x} \Phi_{\delta}=W_{n},
$$

where $W_{n}=\varepsilon_{n k \mu} \partial_{k} V_{\mu}$ is the plastic flow vorticity and $\boldsymbol{\Phi}=\boldsymbol{\Phi}+\boldsymbol{\Phi}^{\prime}$ contains the rotation component of external plastic distortion. Solving (5) in a local coordinate system (normal $\mathbf{n}$, binormal $\mathbf{b}$, tangent $\mathbf{t}$ ) together with the Frenet equation gives the plastic wave velocity proportional to the curvature $\kappa(S, t)$ of a deformed region and directed along the binormals $\mathbf{b}(s, t)$ :

$$
V=\frac{b}{4 \pi} x(s, t) \mathbf{b}(s, t) A(L, d),
$$

where $L$ is the length of a deformed region, $d$ is its lateral dimension, $s$ is the current length, and $\mathbf{b}$ is the Burgers vector. The scale factor is $A=\ln (2 L / d)-1$ for $L \gg d$ and $A=0.2$ for $L \sim d$.

Passing from the motion equation for plastic flow to a nonlinear equation allows us to calculate the curvature $\kappa(S, t)=4 \beta \operatorname{sech}(2 \beta)(S-\alpha t)$, where $\alpha$ and $\beta$ are the torsion and maximum curvature of a deformed region: $\beta$ determines the plastic flux and $\alpha$ gives the rate of its change along $S$.

If we select a global coordinate system such that the $z$ axis is directed along $L$, and $X$ and $E$ are varied in the range of lateral dimensions, we have the following expressions for the strain and rotation tensors, $\varepsilon_{\mu \nu}$ and $\omega_{\mu \nu}$, in the Cartesian coordinate system:

$$
\begin{aligned}
\varepsilon_{z z}=1- & \frac{2}{1+v^{2}} \operatorname{sech}^{2} \xi, \\
\varepsilon_{z x}\left(\omega_{z x}\right) & =\frac{1}{1+v^{2}}\left\{\frac{\alpha b x t}{\pi\left(x^{2}+y^{2}\right)}\left(\frac{1}{\cosh ^{2} \xi}-\frac{1}{\cosh ^{2} \eta}\right) \pm\right. \\
& \left. \pm\left(\frac{\sin (v \xi) \sinh \xi}{\cosh ^{2} \xi}-\frac{v \cos (v \xi)}{\cosh \xi}\right)\right\},
\end{aligned}
$$




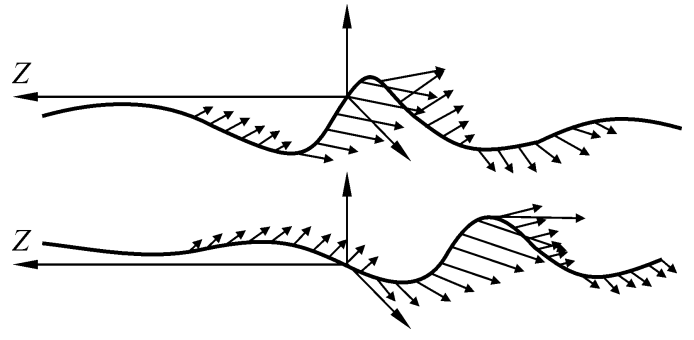

Fig. 1. Plastic wave form and velocity (arrows) in a deformed region

$$
\begin{aligned}
\varepsilon_{z y}\left(\omega_{z y}\right) & =\frac{1}{1+v^{2}}\left\{\frac{\alpha b y t}{\pi\left(x^{2}+y^{2}\right)}\left(\frac{1}{\cosh ^{2} \xi}-\frac{1}{\cosh ^{2} \eta}\right) \pm\right. \\
& \left. \pm\left(\frac{\cos (v \xi) \sinh \xi}{\cosh ^{2} \xi}-\frac{v \sin (v \xi)}{\cosh \xi}\right)\right\} .
\end{aligned}
$$

The subscripts in brackets in the left-hand side of (7) correspond to the rotations $\omega_{\mu \nu}, \xi=2 \beta z+2 \alpha \beta b / \pi A t$, $\eta=\xi-2 \beta z$, and $v=-\alpha / \beta$.

The quantity $\alpha b A / \pi$ represents the plastic wave velocity in the global coordinate system. In the local coordinate system, the wave velocity is determined solely by the torsion $\alpha$.

It should be noted that the scale factor $A$ was initially used to determine the time $t$ in a common coordinate system. This means that the time, being a parameter of plastic deformation, has no absolute meaning and the wave velocity is controlled only by torsion.

Figure 1 shows the form of a plastic strain wave in a deformed region according to (6), (7), with arrows indicating its velocities polarized along the binormals $\mathbf{b}$ perpendicular to the propagation direction.

\subsection{Constitutive equation}

Any deformed region involved in plastic strain localization generates defect and energy flows with attendant heat and mass transfer. In this case, the work function determines the specific entropy production $S$ (dissipative function) and the entropy flux $I$ :

$$
\begin{aligned}
& S=\frac{\left(Q_{i}-J_{i} \mu\right) \partial_{i} T}{T^{2}}-\frac{J_{i} \partial_{i} \mu}{T}-\frac{\partial_{i} T \omega_{i k} v_{k}}{T^{2}}+\frac{\sigma_{k} v_{k}}{T}, \\
& I_{i}=\frac{\omega_{i k} v_{k}}{T}-\frac{Q_{i}}{T}-\frac{\mu J_{i}}{T},
\end{aligned}
$$

where $\omega_{i k}=\varepsilon_{i j k} \alpha_{j}$ on microscale, $\alpha$-strain-induced defect, $\partial_{k} U_{i}-\partial_{i} U_{k}$ on macroscale or antisymmetric component of plastic distortion (rotation mode), $v$ is the plastic strain rate, $\sigma$ is the local stress, $T$ is the temperature at local equilibrium, $\varepsilon_{i j k}$ is the Levi-Civita symbol, $Q_{i}=-\kappa \partial_{i} T / \rho$ is the specific heat flux, $\kappa$ is the heat conductivity, $\rho$ is the material density, $\partial_{i} T$ are the temperature gradient components, $J_{i}=-D \partial_{i} c(z) / \rho$ is the specific mass flux, $D$ is the diffusion coefficient, $\partial_{i} c$ are the concentration gradient components, and $\mu=\mu_{1}-\mu_{2}$ is the difference between the chemical potentials of material and diffusing component.

The first and second terms in the right-hand side of (8) for $S$ represent the entropy production due to heat release and mass transfer; the other two, due to plastic deformation [20]. The former two terms are of second-order infinitesimal with respect to the gradients $\delta_{i} T \delta_{i} T, \delta_{i} T \delta_{i} C$, $\delta_{i} C \delta_{i} \mu$, and the thermoplastic term is of first-order infinitesimal.

The second law of thermodynamics gives us a constitutive equation being the equation of plastic state for a deformed system:

$$
\sigma_{k} v_{k}-\frac{\left(Q_{i}-J_{i} \mu\right) \partial_{i} T}{T}-J_{i} \partial \mu-\frac{\partial_{i} T}{T} \omega_{i k} v_{k} \geq 0 .
$$

It is now evident that the plastic flow supporting this state becomes irreversible. For the second-order terms being small with respect to the gradients, relation (9) is given by

$$
\left(\sigma_{k}-\frac{\partial_{i} T}{T} \omega_{i k}\right) v_{k} \geq 0 .
$$

In the elastic case, the strain and the stress are related by Hooke's law. Likewise, in the plastic case, expressions (9) and (10) relate the macroscale flux, density (translation and rotation modes), and local stress. The deviator $\sigma_{k}$ in (9), (10) is the irreversible stress equal to the difference between the total local stress and its value at the yield point (elastic limit).

Inequalities (9), (10) represent plasticity with work hardening; for perfect plasticity at $v_{k} \neq 0$, an equality is possible. With no temperature gradient, Eq. (10) coincides with the well-known equation of state in plasticity mechanics [26]. Let us dwell on some particular aspects of irreversibility.

Relations (9), (10) suggest that the increment in the work function is positive (Drucker postulate). For unloading $(\sigma<0)$, equality $(10)$ holds only at $v_{k}=0$, implying that unloading proceeds via elastic deformation. Inequality (10) for unloading holds only at $v_{k}<0$ : reverse plastic deformation occurs at total stresses lower than the yield stress (elastic limit), i.e., the process involves softening (Bauschinger effect).

The perfect thermoplastic state with no work hardening, i.e., with (10) equal to zero, corresponds to steady conditions $\left(\partial S / \partial v_{k}=0\right)$ and zero total thermodynamic strength:

$$
\sigma_{k}-\frac{\partial_{i} T}{T} \omega_{i k}=0
$$

This implies that every event of plastic deformation provides conditions for stress relaxation via rotations at gradient temperature.

Creep at ambient temperature results in steady plastic flow when absorbed heat and mass transfer (migration and diffusion) transform into mechanical energy for no change in stress. The constitutive equation of state (11) for these 

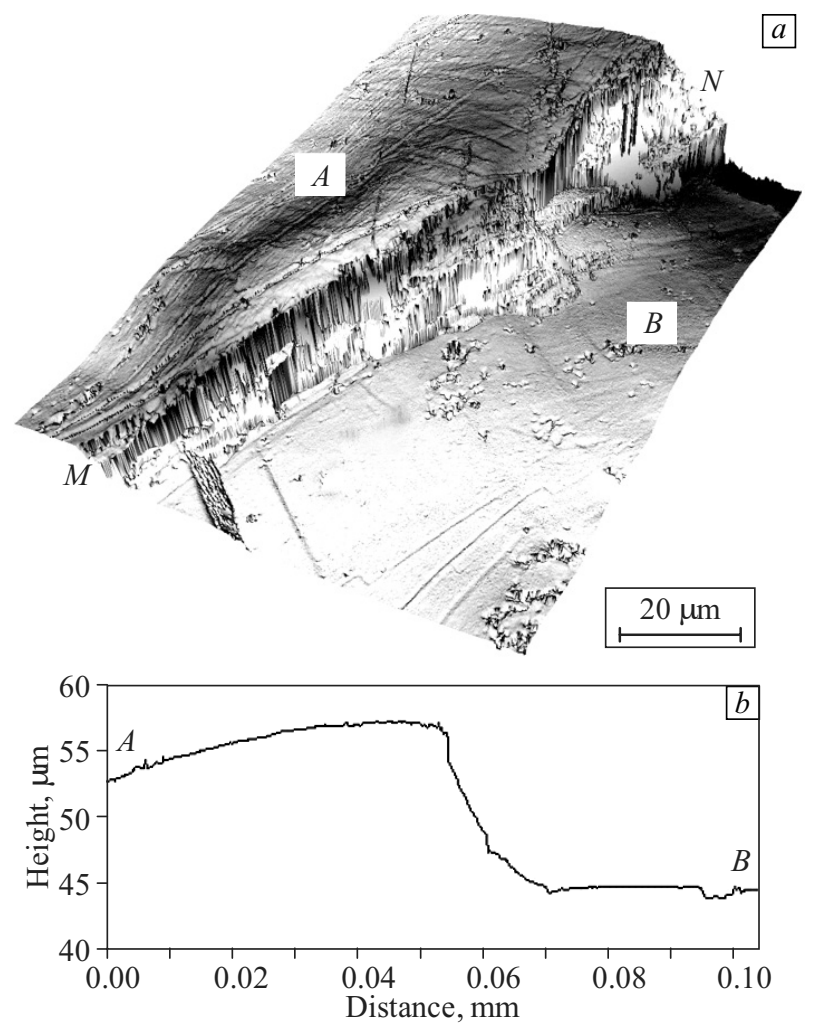

Fig. 2. Denuded grain boundary $M N$ of grains $A$ and $B$ in A999 aluminum under creep at $T=323 \mathrm{~K}, \sigma=11 \mathrm{MPa}$ : interference image (a), surface profile normal to $M N$ (NewView 6200) (b)

conditions represents plastic rotation modes at different creep stages. Let us show it experimentally.

\subsection{Experimental verification of theoretical predictions}

When analyzing three-stage creep in polycrystals, one should allow for a wide range of potential stress sources $\nabla f$. The high rate of creep at its first stage is due to stochastically distributed stress concentrators at triple junctions. At the stage of prefracture, constrained rotations induced by grain boundary sliding create highly nonequilibrium conditions and increase the stress in locally thinned zones (analogue of quasi-necking in cylindrical specimens under uniaxial tension). These potential stress sources are ignored in most creep theories, and the expressions derived above are correct only for the second or steady creep stage.

From relation (11) it follows that the macroscale rotation $\omega_{i k}$ in steady creep must increase with increasing applied stresses $\sigma_{k}$, which is confirmed by experiments.

Figures 2, 3 show grain boundary sliding zones in highpurity A999 Al polycrystals under creep at $\sigma_{1}=11 \mathrm{MPa}$ (Fig. 2) and $\sigma_{2}=18 \mathrm{MPa}$ (Fig. 3). It is seen that although the denuded grain boundary at $\sigma_{1}=11 \mathrm{MPa}$ measures $12 \mu \mathrm{m}$, the accommodating rotation mode of near-boundary deformation is very local and homogeneous. This is quite clear because such conditions provide intragranular rotations via microscale formation and misorientation of a cellular dislocation substructure $[3,4]$. At $\sigma_{2}=18 \mathrm{MPa}$, the denuded grain boundary measures a mere few micrometers but the material involved in plastic rotations near the boundary is extruded through mesoscale displacements of coarse bands. It is this scaling effect of rotation modes that Eq. (11) predicts for creep at increasing applied stresses.

The most severe rotations in A999 Al polycrystals develop at the third creep stage (Fig. 4). Early in tertiary creep, grain boundary sliding causes multiscale fragmentation in near-boundary grain zones. As can be seen in Fig. 4, $a$, grain $C$ is involved in sliding with a highly pronounced tangential component along its denuded boundary (shown by an arrow), which results in complex near-boundary fragmentation. Outer near-boundary layer $A B$ is severely fragmented such that the initial structure degrades. Inner layers $1-3$ are displaced relative to each other with the formation of denuded subboundaries $a b, c d$, ef and cross steps. Thus, the material is fragmented into blocks preserving their translation-invariant structure. It is this invariance that provides $\partial S / \partial v_{k}=0$ in a fragmented material. Noteworthy is that while grain $C$ as a whole rotates clockwise due upward displacements of its zone $B$ (Fig. $4, b$ ), layers $1-3$ are displaced anticlockwise with the formation of blocks. In other words, grain boundary sliding and fragmentation show selfconsistency obeying the angular momentum conservation
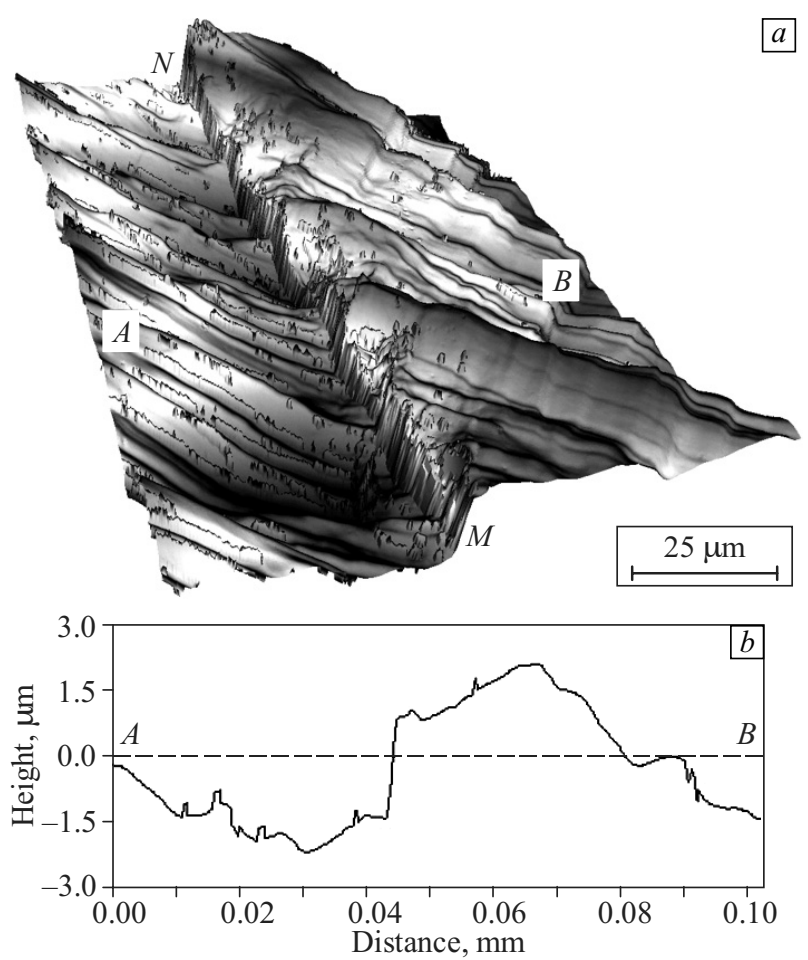

Fig. 3. Extrusion bands along boundary $M N$ of grains $A$ and $B$ in A999 aluminum under creep at $T=323 \mathrm{~K}, \sigma=18 \mathrm{MPa}$ : interference image (a), surface profile normal to $M N$ (NewView 6200) (b) 

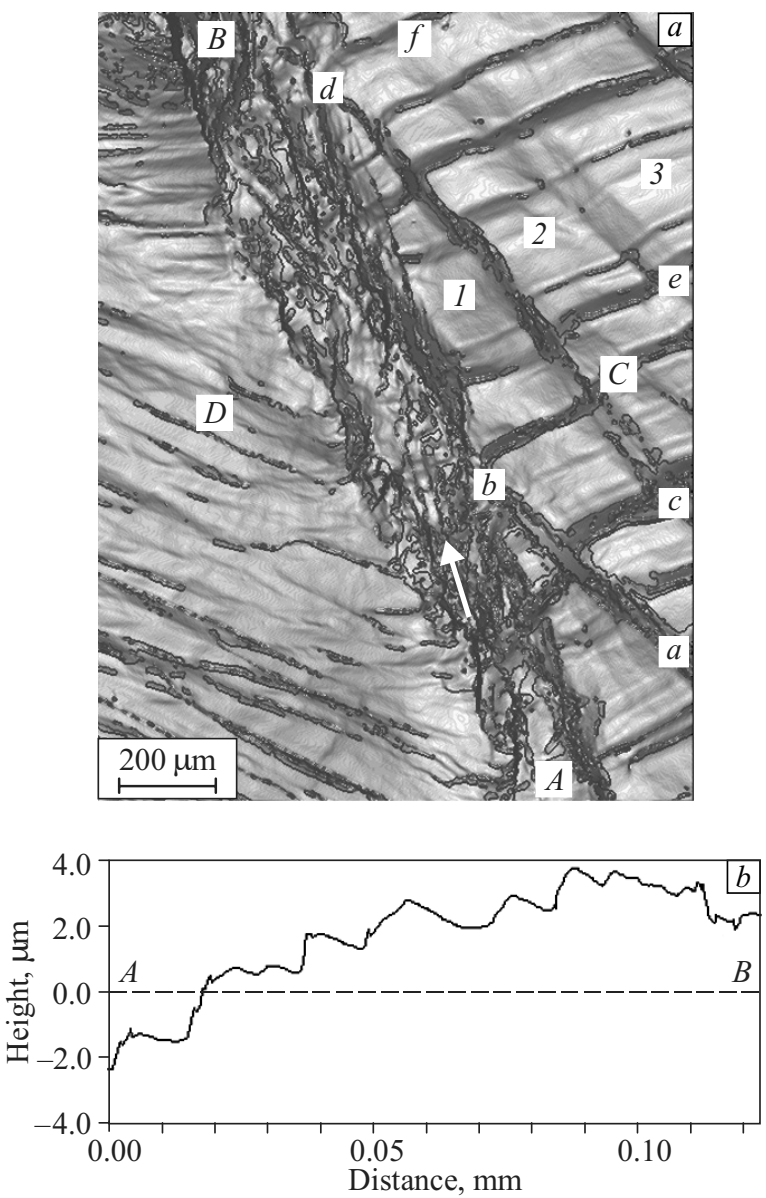

Fig. 4. Extruded mesofragments along boundary $A B$ of grains $C$ and $D$ in A999 aluminum under creep at $T=323 \mathrm{~K}, \sigma=$ $=18 \mathrm{MPa}$ : interference image $(a)$, profile of fragmented mesoband 1 (NewView 6200) $(b)$

law. Cleary, such structural degradation in tertiary creep cannot be described by relations (8)-(11).

Late in the tertiary creep at $\sigma_{2}=18 \mathrm{MPa}$, the surface structure of A999 aluminum reveals a highly nonequilibrium state and multiscale local cross-sectional thinning (quasinecking) indicative of prefracture. It is seen from Fig. 5 that the surface layer in the prefracture zone is involved in rotations via thin mesobands of localized plastic flow. At higher magnification (Fig. 5, b), one can identify that such mesobands propagate stepwise with step heights of about $2 \mu \mathrm{m}$. Mesoband $A B$, which causes quasi-necking before fracture, consists of thin transverse shear bands with steps of $\sim 0.5 \mu \mathrm{m}$. Thus, the material in the quasi-neck zone undergoes submicron fragmentation. Such multiscale fragmentation induced by transverse sliding within mesobands can provide any rotations in a material involved in tertiary creep.

The potential stress sources responsible for quasi-necking before fracture greatly increase the rate of tertiary creep. Because macromechanics is yet unable to account for stochastic potential stress sources at the stages of primary and
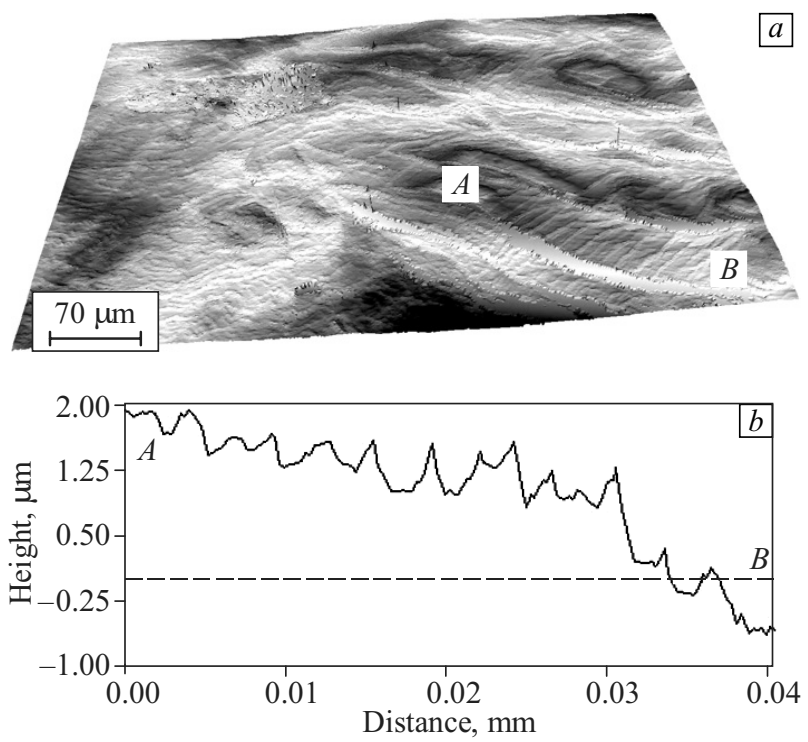

Fig. 5. Transverse bands inside strain localization mesoband $A B$ (quasi-neck region) in A999 aluminum under creep near the point of fracture at $T=323 \mathrm{~K}, \sigma=18 \mathrm{MPa}$ : interference image $(a)$; profile of fragmented mesoband $A B$ (NewView 6200) (b)

tertiary creep, Eqs. (8)-(11) refer to nonlinear mesomechanics of multiscale steady creep.

As has been shown $[3,4]$, polycrystals in tertiary creep undergo delamination in near-boundary intragranular sliding zones (Fig. 6), and this is associated with high lattice curvature and with bifurcations arising at interstices. It is logical to expect that the latter two factors will increase the role of rotation modes in near-boundary intragranular deformation. Let us analyze such rotations in the context of concentration fluctuations $[27,28]$.

\subsection{Concentration fluctuations in translation-rotation plastic flow}

Let us consider concentration fluctuations $\delta c$ in a deformed region at plastic equilibrium when $\Phi \neq 0$ (see Eq. (3)) and write the free energy for them [27]:

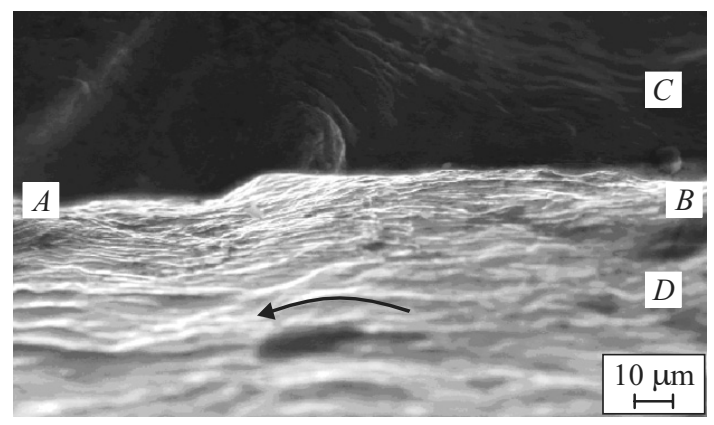

Fig. 6. Noncrystallographic sliding within near-boundary zone of fracture surface as a consequence of grain boundary sliding along boundary $A B$ between grain $C$ and $D$; tertiary creep of $\mathrm{Al}$ polycrystal; scanning electron microscopy 


$$
\begin{aligned}
F & =\int\left\{-a|\delta c|^{2}+\frac{b}{2}|\delta c|^{4}+\right. \\
& \left.+\left|\left(i \partial \mu+\frac{1}{l^{2}} \varepsilon_{\mu \nu k} \Phi_{v} r_{k}\right) \delta c\right|^{2}+\Phi^{2}\right\} \mathrm{d} v .
\end{aligned}
$$

Let $\delta c$ be maximal only along the $z$ axis, i.e., $\partial c / \partial x$, $\partial c / \partial y=\partial c / \partial z$ and $c=c(z)$. In the general case, reasoning that $\delta c$ represents short-wave fluctuations, we have the coefficient $\alpha=\alpha(T)+a^{\prime}\left(q-q_{0}\right)^{2}[27]$ for $\left|q-q_{0}\right|<<q_{0}$. The vector $\mathbf{q}_{0}$ in the reciprocal space corresponds to the vector $\mathbf{l}$ in the straight space, which limits the scale of plastic misorientation $\Phi$ such that $\mathbf{q}_{0} \mathbf{l}=2 \pi$. The $\mathbf{q}$-dependent term in the expression for $\alpha$ is responsible for segregation (weak crystallization) in the region of concentration fluctuations. Because the effect of segregation on superplasticity is slight, we can omit $\alpha^{\prime}\left|q-q_{0}\right|$ and assume the coefficient $\alpha$ to depend only on temperature $\alpha(T)=\tilde{\alpha}\left(T-T^{*}\right)$, where $T^{*}$ is the temperature of stability loss for a high-temperature phase.

Minimization of (12) with respect to $\delta c$ gives

$$
\frac{\partial^{2} \delta c}{\partial^{2} z}-\frac{\partial \delta c}{\partial t}+\delta c-|\delta c|^{2} \delta c=0
$$

where $\partial \delta c / \partial t=v \partial \delta c / \partial z$, and $v=2 u_{z} /\left(a l^{2}\right)$ represents the velocity of fluctuation motion along the $z$ axis and depends on plastic displacements $u_{\mu}=\varepsilon_{\mu v k} \Phi_{v} r_{k}$, temperature $\alpha(T)$, and misorientation scale.

For deriving a relation for concentration fluctuations and plastic flow, we minimize (12) with respect to $u_{\mu}$ for which the parameter $\delta C=|\delta C| e^{i \theta(r)}$ is expressed as the product of its absolute value and argument such that the total velocity of superplastic flow is determined by the phase of nonuniform fluctuations. tem

The resulting equation together with (3) gives the sys-

$$
\begin{aligned}
& \partial_{\mu} \theta=\xi v_{\mu}+\frac{\lambda}{|\delta c|^{2}} \varepsilon_{\mu v k} \partial_{v} \Phi_{k}, \\
& \varepsilon_{\mu \nu k} \partial_{v} \Phi_{k}=V_{\mu}+c_{\mu \nu}^{\beta} p_{\beta}^{v},
\end{aligned}
$$

where $\lambda=b / a, \xi=a l^{2} / 2,|\delta c| \neq 0$.

The above two equations describe the interaction and energy exchange between long-wave plastic fluctuations and short-wave concentration fluctuations. The incompatibility of Eqs. (14) and (15) provides low rates of steady creep. High-rate rotations in grain boundary sliding cannot be immediately accommodated by microscale rotations of intragranular dislocation glide, as is required by the angular momentum conservation law. Because dislocations are generated by structural transformation flows in the planar subsystem of a solid (surface layers and all internal interfaces, including grain boundaries) [14, 15], there should be a tangential displacement component in grain boundary sliding and a sinusoidal normal stress distribution at grain boundaries [29]. In the grain boundary zones of tensile normal stress, clusters of positive ions arise and generate dislocation cores in the zones of intragranular sliding. The rotation mode of dislocation deformation develops slowly, and this greatly decreases the grain boundary sliding velocity.

Equations (14) and (15) show compatibility for tertiary creep when high lattice curvature and high concentrations of interstitial states arise in near-boundary grain zones. In this case, the concentration fluctuations described by (14) occur both at grain boundaries and in near-boundary intragranular sliding zones. The velocities $v_{\mu}$ and $V$ take very high values of the same order. The rotation modes of grain boundary sliding are fully accommodated by the concentration fluctuations in near-boundary intragranular sliding zones where the material structure is delaminated and degenerated. Such an effect was observed earlier $[3,4]$ but had no explanation at that time (Fig. 6).

The energy exchange between grain boundary sliding and concentration fluctuations in near-boundary plastic flow is most efficient under resonance conditions, with $v_{\mu}$ and $V_{\mu}$ having the same order. In this case, collective motion provides displacements of an area equal in size to a concentration kink, i.e., Eq. (13) has the solution

$$
\delta c: \pm \operatorname{th}\left(\frac{1}{2} \frac{z-v t}{\sqrt{1-v^{2}}}\right),
$$

where $v$ and $t$ are determined after solving (13).

Combination of (3) and (14) gives the velocity of plastic flow for tertiary creep:

$$
\partial_{\mu} \theta=\frac{\lambda}{|\delta c|^{2}} V_{\mu}+\xi_{v_{\mu}}+\frac{\lambda}{|\delta c|^{2}} c_{\mu \nu}^{\beta} p_{\beta}^{v} .
$$

The first term in the right-hand side of (17) represents the velocity of plastic wave flow (6) increased by a factor of $\lambda /|\delta c|^{2}$.

The quantity $V_{\mu}$ was calculated with regard to the rotation component of plastic distortion $\Phi^{\prime}$ and plastic displacement $u_{\mu}$. Thus, the term $\xi_{v_{\mu}}$ in the right-hand side of (17) is determined by the gradient of external sources, and the next term by delayed polarization when $V_{\mu}$ reaches its maximum value. At $c_{\mu \nu}^{\beta} \rightarrow 0$ in a deformed region, the last term of (17) is responsible only for near-boundary intragranular deformation. The rate of tertiary creep calculated by (17) is in good agreement with experimental results.

\section{Discussion}

The necessity of accounting for lattice curvature in a deformed solid is pointed out in many papers [11-18]. For example, high lattice curvature is responsible for shear banding and plastic distortion in surface-hydrogenated $\mathrm{Ti}$ and commercial Al with a thin A999 Al film under alternate cyclic bending [17] and for dynamic rotations in metal surface layers during shock wave propagation [10] and opening mode cracking $[14,15]$. These effects of lattice curvature suggest that its zones develop translation-rotation de- 


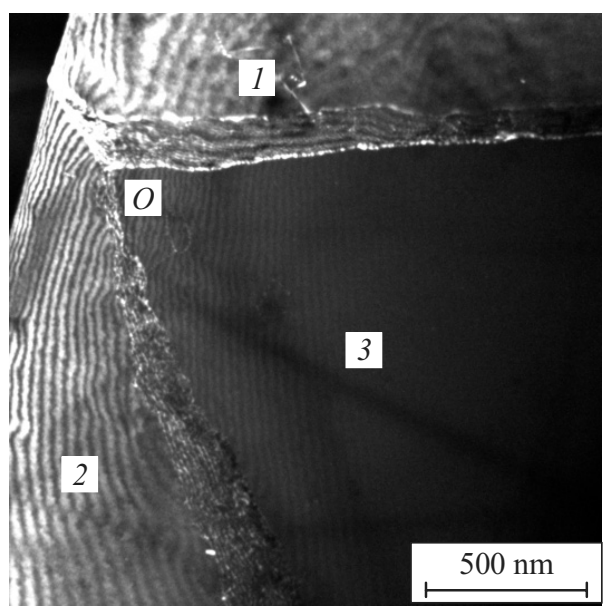

Fig. 7. TEM image showing extinction contours in near-boundary zones with high lattice curvature and structural degradation in polycrystalline aluminum; tertiary creep at $T=50^{\circ} \mathrm{C}$

fects, which are not considered by conventional theories in which the lattice is taken as translation-invariant.

The lattice curvature $\chi$ induces a counter stress field $\sigma_{z z}$ and is expressed as [30]:

$$
\chi=4 \beta \operatorname{sech}[2 \beta(s+4 v t)], \beta=\frac{\sigma_{z z}}{d E},
$$

where $d$ is the width of a curved plane, $s$ is the current coordinate, $t$ is time, and $v$ is the velocity of local structural transformation in the curved plane in a local coordinate system. From expression (18) it follows that translationrotation defects should arise in a deformed solid, providing relaxation of the stress field $\sigma_{z z}$ and transformation of the elastic curvature into plastic rotation.

When the pair interaction potential has a single minimum, the particle system potential can take bifurcation minima due to increasing interatomic spacing $[12,13]$ such that atoms can pass from lattice sites to interstices, forming "translation-rotation" strain-induced defects and providing noncrystallographic plastic flow. Such strain-induced defects arise in all deformed solids but their identification is easiest in polycrystals under creep which reveal highly pronounced accommodation of their primary grain boundary sliding by translation-rotation plastic flow in near-boundary intragranular regions. Let us consider some experimental evidences for the generation of rotation defects in tertiary creep (Figs. 7, 8).

Figure 7 shows TEM image with extinction contours demonstrating the presence of high lattice curvature which is equal to $25^{\circ} \mu \mathrm{m}^{-1}$ in a near-boundary region. The sliding velocities $V$ and $v_{\mu}$, in this case, are of the same order, and the result is an abrupt increase in the rate of tertiary creep.

Very important data on the formation mechanism of subboundaries in grains at the stage of tertiary creep is represented in Fig. 8. Early in the formation, the structure of subboundaries points to their shear band nature having a

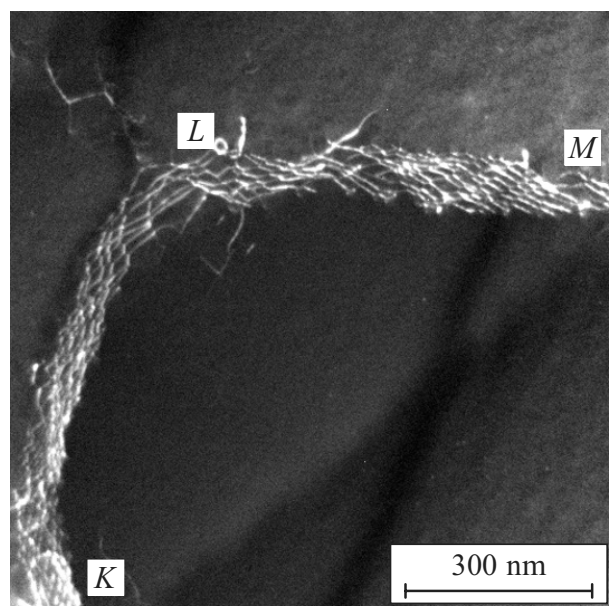

Fig. 8. TEM image of subboundary $K L M$ formed during submicron fragmentation in polycrystalline aluminum; tertiary creep at $T=50^{\circ} \mathrm{C}$

lot of the short local noncrystallographic shears within the high lattice curvature region (Fig. 8, fragment $L M$ ). At the second stage, the shear band makes a sharp turn and form a fiber structure (Fig. 8, fragment $K L$ ) which greatly contributes to low-angle misorientations of subgrains in tertiary creep. This type of strain-induced defects should be introduced to describe nonlinear plastic deformation and fracture in tertiary creep $[10,17]$.

\section{Conclusions}

Thus, the developed nonlinear theory of micro-, mesoand macroscale plastic flow gives a constitutive equation for the velocity of plastic flow, rotation modes, local irreversible stress, temperature, heat and mass flux densities with regard to structural transformations. The theory agrees well with experimental data on multiscale translation-rotation modes in Al polycrystals under creep. The results suggest that such modes of intragranular plastic flow are initiated by grain boundary sliding as a primary process at all creep stages. Slow intragranular rotations provide constant rates of steady creep, and very high rates of tertiary creep are provided by high lattice curvature and numerous nonequilibrium interstices that arise in near-boundary regions due to grain boundary sliding. The structure of a material thus transforms through noncrystallographic sliding in local high curvature zones up to the point of plastic collapse and fracture.

\section{Acknowledgments}

The work was performed under Fundamental Research Program of the State Academies of Sciences of Russia for 2013-2020 and supported by the International Research Staff Exchange Scheme under 7th EU Framework Program (grant No. 612552), Russian Foundation for Basic Research 
(project No. N170100691), and President of the Russian Federation (grant for support of leading scientific schools No. NSh-10186.2016.1).

\section{References}

1. Kassner M.E. Fundamentals of Creep in Metals and Alloys. - Oxford: Elsevier Science, 2009.

2. Gifkins R.C. Grain-boundary sliding and its accommodation during creep and superplasticity // Met. Trans. A. -1976 . - V. 7. - No. 8. P. $1225-1232$.

3. Panin V.E., Elsukova T.F., Surikova N.S., Popkova Yu.F., Borisyuk D.V. The role of rotation modes of plastic deformation in failure of highpurity $\mathrm{Al}$ at ambient temperature creep // Deform. Razrush. Mater. 2016. - No. 12. - P. 2-9.

4. Panin V.E., Egorushkin V.E., Panin A.V., Chernyavskii A.G. Plastic distortion as a fundamental mechanism in the nonlinear mesomechanics of plastic deformation and fracture // Phys. Mesomech. - 2016. V. 19. - No. 3. - P. $255-268$

5. Wadsworth J., Ruano O.A., Sherby O.D. Denuded zones, diffusional creep and grain boundary sliding // Metall. Mater. Trans. A. - 2002. V. 33. - P. 219-229.

6. Matsunaga T., Kameyama T., Sato E. Grain boundary sliding induced by lattice dislocation activity during ambient temperature creep in hcp metals // IOP Conf. Mater. Sci. Eng. - 2009. - V. 3. - Article 012014.

7. Matsunaga T., Kameyama T., Ueda S., Sato E. Grain boundary sliding during ambient-temperature creep in hexagonal close-packed metals // Phil. Mag. - 2010. - V. 90(30). - P. 4041-4054.

8. Ruano O.A., Sherby O.D., Wadsworth J., Wolfenstine J. Diffusional creep and diffusion-controlled dislocation creep and their relation to denuded zones in $\mathrm{Mg}-\mathrm{ZrH}_{2}$ materials // Scripta Mater. - 1998. V. 38. - P. 1307-1314.

9. Wilshire B., Burt $H$. Tertiary creep of metals and alloys // Int. J. Mater. Res. Adv. Tech. - 2005. - V. 96. - No. 6. - P. 552-557.

10. Panin V.E., Egorushkin V.E., Elsukova T.F., Surikova N.S., Pochivalov Yu.I., Panin A.V. Multiscale Translation-Rotation Plastic Flow in Polycrystals. Handbook of Mechanics of Materials / Ed. by C.-H. Hsuch et al. - Singapore: Springer Nature, 2018. - doi 10.1007/978-981-106855-3 77-1.

11. Myasnikov V.P., Guzev M.A. A geometrical model of the defect structure of an elastoplastic continuous medium // J. Appl. Mech. Tech. Phys. - 1999. - V. 40. - No. 2. - P. 331-340.

12. Guzev M.A. Structure of displacement and stress fields in a nonEuclidean continuum model // Solid Mechanics: Nizhny Novgorod State Univ. Vestnik. - 2011. - V. 4. - P. 1461-1462.
13. Guzev M.A., Dmitriev A.A. Bifurcational behaviour of potential energy in a particle system // Phys. Mesomech. - 2013. - V. 16. - No. 4. P. $287-293$.

14. Moiseenko D.D., Panin S.V., Maksimov P.V., Panin V.E., Babich D.S., Berto F. Computer simulation of material behaviour at the notch tip: Effect of microrotations on elastic energy release // AIP Conf. Proc. 2016. - V. 1783. - P. 020157. - doi 10.1063/1.4966450.

15. Panin V.E., Grinyaev Yu.V., Egorushkin V.E. Foundations of physical mesomechanics of structurally inhomogeneous media // Mech. Solids. $-2010 .-$ V. 45. - No. 4. - P. 501-518.

16. Panin V.E., Egorushkin V.E., Panin A.V. Nonlinear wave processes in a deformable solids as a multiscale hierarchically organized system // Phys. Usp. - 2012. - V. 55(12). - P. 1260-1267.

17. Panin V.E., Egorushkin V.E., Surikova N.S., Pochivalov Yu.I. Shear bands as translation-rotation mode of plastic deformation in solids under alternate bending // Mater. Sci. Eng. A. - 2017. - V. 703. P. $451-460$.

18. Tyumentsev A.N., Ditenberg I.A., Korotaev A.D., Denisov K.I. Lattice curvature evolution in metal materials on meso- and nanostructural scale of plastic deformation // Phys. Mesomech. - 2013. - V. 16. No. 4. - P. 319-334.

19. Egorushkin V.E. Gauge dynamic theory of defects in nonuniformly deformed media with structure. Interface behavior // Russ. Phys. J. 1990. - V. 33. - P. 135-149.

20. Egorushkin V.E. Dynamics of plastic deformation: Waves of localized plastic deformation in solids // Russ. Phys. J. - 1992. - V. 35. P. 316-334.

21. Kroner E. Gauge Field Theories of Defects in Solids. - Stuttgart: Max. Planck. Inst., 1982.

22. Kadič A., Edelen D. G.B. Gauge Theory of Dislocations and Disclinations: Lecture Notes. - Heidelberg: Springer-Verlag, 1983.

23. Edelen D. G.B., Lagoudas D.C. Gauge Theory and Defects in Solids. Amsterdam: North Holland Publ., 1988.

24. Sanchez-Palencia E. Non-Homogeneous Media and Vibration Theory: Lecture Notes in Physics. - Berlin: Springer, 1982.

25. Kosevich A.V. Dynamical theory of dislocations // Sov. Phys. Usp. 1965. - V. 7. - P. 579-609.

26. Ziegler H. Some Extremum Principles in Irreversible Thermodynamics with Application to Continuum Mechanics // Progress in Solid Mechanics. - Amsterdam: North Holland, 1963.

27. Lebedev V.V. Fluctuation Effects in Macrophysics. Moscow: MTsNMO, 2004.

28. Patashinsky A.Z., Pokrovsky V.L. Fluctuation Theory of Phase Transitions. - Moscow: Nauka, 1982.

29. Cherepanov G.P. On the theory of thermal stresses in thin bounding layer // J. Appl. Phys. - 1995. - V. 78. - P. 6826-6832.

30. Landau L.D., Lifshits E.M. Theory of Elasticity. - Moscow: Nauka, 1987. 